\title{
Loss of serum response factor in mature neurons in the dentate gyrus alters the morphology of dendritic spines and hippocampus-dependent behavioral tasks
}

\author{
Karolina Nader $^{1} \cdot$ Anna Krysiak ${ }^{1} \cdot$ Anna Beroun ${ }^{1} \cdot$ Martyna Pekala $^{1} \cdot$ Magda Szymanska ${ }^{1} \cdot$ Bozena Kuzniewska $^{1}$. \\ Kasia Radwanska² $\cdot$ Leszek Kaczmarek ${ }^{1} \cdot$ Katarzyna Kalita $^{1}$
}

Received: 17 January 2019 / Accepted: 19 July 2019 / Published online: 2 August 2019

(C) The Author(s) 2019

\begin{abstract}
Serum response factor (SRF) is a major transcription factor that regulates the expression of several plasticity-associated genes in the brain. Although the developmental expression of SRF in excitatory neurons is crucial for establishing proper hippocampal circuitry, no substantial evidence of its role in unstimulated mature neurons has been provided. The present study used time-controlled, conditional SRF knockout mice and found that the lack of SRF in adult neurons led to decreased actin levels and inactivation of the actin-severing protein cofilin 1 through its increase in phosphorylation at Ser3. The augmentation of cofilin 1 phosphorylation correlated with an alteration of dendritic spine morphology in the dentate gyrus, which was reflected by an increase in the number of spines that clustered into the long-spine category. The changes in spine morphology coincided with a lower amplitude and frequency of miniature excitatory postsynaptic currents. Moreover, SRF knockout animals were hyperactive and exhibited impairments in hippocampus-dependent behaviors, such as digging, marble burying, and nesting. Altogether, our data indicate that the adult deletion of neuronal SRF leads to alterations of spine morphology and function and hippocampus-dependent behaviors. Thus, SRF deletion in adult neurons recapitulates some aspects of morphological, electrophysiological, and behavioral changes that are observed in such psychiatric disorders as schizophrenia and autism spectrum disorders.
\end{abstract}

Keywords Serum response factor $\cdot$ Cofilin $1 \cdot$ Dentate gyrus $\cdot$ Structural plasticity $\cdot$ Rodent-specific behaviors

\section{Introduction}

Serum response factor (SRF) is a transcription factor that is highly expressed in granular neurons in the dentate gyrus (DG) of the hippocampus (Etkin et al. 2006; Ramanan et al. 2005). It regulates the expression of many cytoskeletal genes and genes that are activated by neuronal stimulation

Karolina Nader and Anna Krysiak contributed equally to this manuscript.

Katarzyna Kalita

k.kalita@nencki.gov.pl

1 Laboratory of Neurobiology, Nencki Institute of Experimental Biology, Polish Academy of Sciences, 3 Pasteur Street, 02-093 Warsaw, Poland

2 Laboratory of Molecular Basis of Behavior, Nencki Institute of Experimental Biology, Polish Academy of Sciences, 3 Pasteur Street, 02-093 Warsaw, Poland
(Benito et al. 2011; Etkin et al. 2006; Kalita et al. 2006; Kuzniewska et al. 2016; Losing et al. 2017; Miano et al. 2007; Parkitna et al. 2010; Ramanan et al. 2005; Treisman 1995). During brain development, SRF-regulated genes control the neuronal cell migration, neurite outgrowth and organization of the DG and the formation of mossy fiber circuitry (Alberti et al. 2005; Etkin et al. 2006; Knoll et al. 2006; Lu and Ramanan 2011; Scandaglia et al. 2015; Stritt and Knoll 2010). The early postnatal deletion of $S r f$ resulted in impairments in long-term potentiation and long-term depression and alterations of the formation of immediate memory of a novel context (Etkin et al. 2006; Ramanan et al. 2005). In contrast to early SRF knockout (KO), time-specific SRF deletion in adult excitatory neurons does not influence gross morphology of the hippocampus (Kuzniewska et al. 2016; Losing et al. 2017). Moreover, SRF deletion in the mature brain caused a very limited alteration of basal gene expression, restricted mostly to genes that encode the actin cytoskeleton (Kuzniewska et al. 2016; Losing et al. 2017; 
Parkitna et al. 2010). Nevertheless, these mice exhibit hyperactivity, a decrease in anxiety-like behavior, and epilepsy (Kuzniewska et al. 2016; Losing et al. 2017; Zimprich et al. 2017).

The DG is part of the hippocampal formation and mostly composed of granule cells (GCs) (Kempermann et al. 2004; Radic et al. 2017). The DG receives excitatory inputs from the entorhinal cortex, and GCs send excitatory outputs via mossy fibers to pyramidal cells in the Cornu Ammonis CA3 (Amaral et al. 2007). Alterations of hippocampal DG circuity have recently been implicated in the pathophysiological mechanisms of psychiatric disorders (Hagihara et al. 2013; Yu et al. 2014).

To investigate whether SRF controls the morphology and function of mature GCs, we employed a model of SRF depletion that was restricted to excitatory neurons (Kuzniewska et al. 2016). In the present study, we found that the ablation of SRF in adult GCs decreased $\beta$-actin levels and led to cofilin 1 inactivation through an increase in the phosphorylation at regulatory Ser3. The decrease in actin expression coincided with abnormal spine morphology and impairments in basal excitatory synaptic transmission in DG neurons. Moreover, the lack of SRF increased animal activity and impaired rodent-typical behaviors, such as digging, marble burying, and nesting. Altogether, our data indicate that SRF expressed in adult excitatory neurons plays a crucial role in maintaining actin expression and controlling the morphological and electrophysiological properties of granular neurons and innate animal behaviors.

\section{Materials and methods}

\section{Animals}

Conditional Srf KO mice (mutant mice; $\mathrm{Srf} / \mathrm{f} / \mathrm{CaMKCreERT2}$ ) were generated as previously described (Kuzniewska et al. 2016). As a control, CreERT2-negative littermates were used $\left(S r f^{f / f}\right.$; control mice; wild type [WT]). The mice were bred on a C57BL/6J genetic background. Adult male and female $\mathrm{Sr}^{\mathrm{f} / \mathrm{f}}$ and $\mathrm{Sr} f^{\mathrm{f} / \mathrm{fCAMKCreERT2}}$ mice were intraperitoneally injected with $1 \mathrm{mg}$ tamoxifen (TAM; cat. \#T5648, Sigma) twice daily for 10 days, resulting in the translocation of Crerecombinase to the nucleus. Experiments were performed at least 6 weeks after the TAM injection. The animals were bred in the Animal House of the Nencki Institute of Experimental Biology. The mice were housed in individual cages with a $12 \mathrm{~h} / 12 \mathrm{~h}$ light/dark cycle, constant temperature $\left(23-25^{\circ} \mathrm{C}\right)$, and food and water available ad libitum. Both male and female mice were used for the experiments, except when marked otherwise. All of the experiments were performed by experimenters who were blind to mouse genotype. All of the work was conducted in accordance with the European Community Council Directive (86/609/EEC) and Animal Protection Act of Poland (Directive 2010/63/ EU). The procedures were approved by the 1st Local Ethics Committee in Warsaw, Poland (permission no. 389/2012, 678/2015, and 144/2016).

\section{RNA preparation and quantitative real-time polymerase chain reaction}

Total RNA was isolated from the mouse hippocampus using the RNeasy Mini Kit (cat. \#74104; Qiagen) as described by the manufacturer. DNA contamination was removed by digestion with DNase I (cat. \#1023460, Qiagen). The RNA concentration was calculated from absorbance at $260 \mathrm{~nm}$, and the 260/280 $\mathrm{nm}$ absorbance ratio determined the purity of RNA. RNA was reverse transcribed with SuperScript III or IV Reverse Transcriptase (cat. \#18080-044, \#18090050, Invitrogen) according to the manufacturer's instructions. Quantitative real-time polymerase chain reaction (PCR) was performed using Fast TaqMan Master Mix (cat. \#44456, Applied Biosystems) with an Applied Biosystems 7900HT Fast Real-Time PCR System using TaqMan probes (ThermoFisher). Fold changes in expression were determined using the $\Delta \Delta \mathrm{C}_{\mathrm{T}}$ relative quantification method. The values were normalized to relative amounts of GAPDH.

\section{Immunohistochemistry}

The animals were perfused with $4 \%$ paraformaldehyde (PFA). Brains were then fixed in $4 \%$ PFA overnight at $4{ }^{\circ} \mathrm{C}$ and cut into $40-\mu \mathrm{m}$ coronal slices. For SRF staining, brain slices were stained with anti-SRF antibody (1:500; cat. \#sc13029; Santa Cruz Biotechnology) overnight at $4{ }^{\circ} \mathrm{C}$ followed by avidin/biotin complex (cat. \#PK-6100, Vector) and visualized using SIGMAFAST ${ }^{\mathrm{TM}}$ DAB (cat. \#D0426, Sigma). For Nissl staining, the brain sections were stained with $0.1 \%$ cresyl violet solution and $3 \%$ acetic acid for $5 \mathrm{~min}$, washed, dehydrated, cleared in xylene, and coverslipped.

\section{Western blot}

Twenty micrograms of protein extracts were run on polyacrylamide gels (cat. \#4569033, BioRad). The standard procedure of Western blot was performed using anti-Ser 3-phospho cofilin 1 and cofilin 1 (cat. \#3313, \#5175, Cell Signaling), and $\beta$-actin (cat. \#A1978, Sigma) antibodies. To ensure equal total proteins level, blots were reprobed with tubulin (cat. \#T9026, Sigma) antibodies. For detection, the chemiluminescent method was used. To quantify individual bands, a scan of photographic films was analyzed by densitometry with GeneTools Software (Syngene). 


\section{Electrophysiology}

Whole-cell patch-clamp recordings were carried out in voltage-clamp mode. Mice (male and female) were anesthetized with isoflurane and decapitated. Brain slices (coronal, $250 \mu \mathrm{m}$ thick) prepared using Leica VT 1200S vibratome in ice-cold NMDG solution (135 mM NMDG, $1.2 \mathrm{mM} \mathrm{KH}_{2} \mathrm{PO}_{4}, 1 \mathrm{mM} \mathrm{KCl}, 1.5 \mathrm{mM} \mathrm{MgCl} 2 \cdot 6 \mathrm{H}_{2} \mathrm{O}, 0.5 \mathrm{mM}$ $\mathrm{CaCl}_{2} \cdot 2 \mathrm{H}_{2} \mathrm{O}, 20 \mathrm{mM}$ choline bicarbonate, $10 \mathrm{mM}$ D-glucose, saturated with carbogen $-95 \% \mathrm{O}_{2}, 5 \% \mathrm{CO}_{2}$ ) were then transferred to the artificial cerebrospinal fluid (ACSF) solution $\left(119 \mathrm{mM} \mathrm{NaCl}, 2.5 \mathrm{mM} \mathrm{KCl}, 1.3 \mathrm{mM} \mathrm{MgCl} 2 \cdot 6 \mathrm{H}_{2} \mathrm{O}, 1 \mathrm{mM}\right.$ $\mathrm{NaH}_{2} \mathrm{PO}_{4}, 26 \mathrm{mM} \mathrm{NaHCO}, 20 \mathrm{mM}$ D-glucose, $2.5 \mathrm{mM}$ $\mathrm{CaCl}_{2} \cdot 2 \mathrm{H}_{2} \mathrm{O}$, saturated with carbogen), incubated for $12 \mathrm{~min}$ at $31{ }^{\circ} \mathrm{C}$ and then at least $1 \mathrm{~h}$ at room temperature. During recordings, slices were held in a recording chamber perfused with carbogenated ACSF solution supplemented with $100 \mu \mathrm{M}$ picrotoxin and $0.5 \mu \mathrm{M}$ tetrodotoxin and heated up to $31^{\circ} \mathrm{C}$. Granule cells of the upper blade of dentate gyrus were identified visually. Borosilicate glass capillaries (4-7 M $\Omega$ resistance) used for patch-clamp recordings were filled with Cs-based internal solution (130 mM Csgluconate, $20 \mathrm{mM}$ HEPES, $0.4 \mathrm{mM}$ EGTA, $3 \mathrm{mM}$ TEACl, 4 mM Na 2 ATP, 0.3 mM NaGTP, 4 mM QX-314Cl; osmolarity: 285-290 mOsm, $\mathrm{pH}=7.0-7.1$ ). Data were acquired using custom algorithms in Igor Pro (Wavemetrics) with an NPI amplifier and digitized at $10 \mathrm{kHz}$ with an ITC-18 InstruTECH/HEKA. Recorded currents were filtered at $2 \mathrm{kHz}$. Series and input resistances were monitored throughout the experiment. To measure miniature excitatory postsynaptic currents (mEPSCs) 10- to 20-min-long voltage-clamp recordings were collected. Miniature events were analyzed using the MiniAnalysis software (Synaptosoft). mEPSCs amplitude detection threshold was set to 7 pA. All mini events automatically detected by the software were verified visually by the experimenter.

\section{Analysis of dendritic spine morphology}

Serum response factor $\mathrm{KO}$ and WT mice (male and female $n=8$ ) were anesthetized and transcardially perfused with phosphate-buffered saline (PBS) and with 1.5\% paraformaldehyde (PFA) at room temperature. The brains were placed in $1.5 \%$ PFA for 20 min for postfix and then transferred to ice-cold PBS for at least $20 \mathrm{~min}$. Next, these brains were cut on a vibratome into $130-\mu \mathrm{m}$-thick slices and placed in PBS at room temperature for $1 \mathrm{~h}$. Gene gun technique was used to label sections with tungsten particles (Bio-Rad, Hercules, CA, USA) coated with lipophilic dye DiI (1,1'-dioctadecyl3,3,3',3'-tetramethyl indocarbocyanine perchlorate; d-3911, cat. \#D282, ThermoFisher). The slices were incubated in PBS at the room temperature for about $3 \mathrm{~h}$ and next in 1.5\% PFA in $4{ }^{\circ} \mathrm{C}$ overnight, which enables diffusion of the dye into neuronal processes and allows to visualize dendritic spines. Z-stacks of confocal images of the seven dendrites per animal from the middle molecular layer of the upper blade of dorsal DG were acquired with 561-nm laser line with Zeiss LSM780 confocal system. Neurons of immature morphology (very sparse dendritic spines) were excluded from the analysis. The semiautomatic SpineMagick! software was used to measure and analyze dendritic spines by obtaining the virtual skeletons (Ruszczycki et al. 2012). We used a scale-free parameter (the length/width ratio) which reflect the spine shape (Michaluk et al. 2011). Length/width ratio was calculated and plotted using a logarithmic scale. The density of spines was calculated as a number of spines per $1 \mu \mathrm{m}$ of dendrite length. Spines' shapes were divided into clusters and then sorted into two groups: "long" and "mushroom and stubby" spines using custom scripts (Jasinska et al. 2016).

\section{Behavioral tests}

Open field The apparatus had a wooden floor $(50 \mathrm{~cm} \times 50 \mathrm{~cm})$ surrounded by walls $(34 \mathrm{~cm}$ hight). Animals' behavior was monitored by a video camera placed above the center of the apparatus. Adult females (WT $n=12$, SRF KO $n=12$, about 6 months old or older) were put individually in one corner of the open field facing the wall and were allowed to explore freely for $15 \mathrm{~min}$. The floor of the apparatus was cleaned with 5\% ethanol after each session. Data were analyzed using EthoVision 3.1 System (Noldus Information Technology), total distance traveled was counted.

Species-typical behaviors All species-typical behaviors tests were performed on the same group of animals (WT $n=10$, SRF KO $n=7$; adult female and male, about 6 months old or older), starting from overnight nesting test and then marble burying and digging.

Nesting Mice were housed individually in their home cages with standard bedding for at least 1 week. After that time paper towel (divided into six pieces $9 \mathrm{~cm} \times 9 \mathrm{~cm}$ each) was placed in the middle of each cage and left overnight to assess the nest building ability of the mice. The following scoring system was used: (1) paper towel was mostly untouched (>90\%) and was left in the middle of the cage; (2) flat pad-shaped nest, mostly moved to a corner; (3) more complex nest with biting the towel but not gathered in one place; (4) nest with shredded paper to form a cup; (5) perfect nest, paper towel torn up to form a crater, where walls are higher than mouse body height. Nest building is spontaneous home cage behavior of mice. It indicates healthy functioning and well-being of an animal (Jirkof 2014; Moretti et al. 2005). It is also interpreted by some authors as social behavior (Crawley 2012).

Marble burying Clean cage was filled $5 \mathrm{~cm}$ deep with bedding, tamped down to make even and flat surface. The 
same 12 glass marbles (15 $\mathrm{mm}$ in diameter) were placed in a regular pattern in two rows on bedding surface. Mice were placed individually into the experimental cage for $30 \mathrm{~min}$. After that time, mice were taken to their home cages, and the number of marbles buried to at least two-thirds of their depth with bedding was counted. The same cage was re-used for all tested animals.

Digging Clean cage was prepared with the flat surface of wooden, 5 -cm-deep bedding. Mice were placed individually in the experimental cage. The duration of the test was $3 \mathrm{~min}$ and latency to start digging, a number of digging bouts and total duration of digging were recorded and analyzed. Coordinated movements of force and/or hindlimbs which displace the wooden bedding defined the digging. The new bedding was used for all tested animals. Marble burying measures spontaneous digging behavior, which is typical for mice and is dependent on hippocampal function (Deacon 2006; Deacon and Rawlins 2005).

\section{Statistical analyses}

To compare the distributions, Shapiro-Wilk normality test was performed. To test the differences between two groups, unpaired $t$ test or Mann-Whitney test (nonparametric) was used. When required repeated-measures ANOVA was performed, followed by Bonferroni's multiple comparisons post hoc test. To compare the cumulative distributions of mEPSCs amplitudes and frequencies, the Kolmogorov-Smirnov test was used. The number of animals and neurons which were used for analysis is provided in figures legend. Data on the graphs are expressed as cumulative probability or means \pm standard errors of the means (SEM). The difference between the experimental groups was considered as significant when $p<0.05$. Results were analyzed in GraphPad Prism software.

\section{Results}

\section{Decrease in $\beta$-actin expression in hippocampal neurons in SRF KO animals}

The genome-wide analysis of basal gene expression in animals with the adult deletion of neuronal SRF showed changes in mRNA that were restricted to genes that encode the actin cytoskeleton, such as Actb and Actgl (Kuzniewska et al. 2016; Losing et al. 2017; Parkitna et al. 2010). To confirm the downregulation of actin expression in the hippocampus in SRF KO animals, we used inducible conditional $\operatorname{Srf} \mathrm{KO}$ mice to ablate $\operatorname{Srf}$ exclusively in adult, excitatory forebrain neurons (Erdmann et al. 2007; Kuzniewska et al. 2016). Actb, Actgl, and Actal mRNA levels were analyzed in the hippocampus in mutant and control littermates using quantitative real-time PCR. We found decreases in the levels of mRNA of $\beta$-actin and non-muscle $\gamma$-actin in the hippocampus in SRF KO animals, but not in smooth muscle $\alpha$-actin (Fig. 1a).

To assess the consequences of SRF depletion in adult neurons, we focused on the morphology of the hippocampus. Using Nissl staining, we confirmed the lack of gross neuroanatomical defects in SRF KO animals with low SRF expression in the hippocampus (Fig. 1c) (Kuzniewska et al. 2016). We also analyzed the size of the hippocampal formation in adult WT and SRF KO animals. No differences were found in the total weight of the hippocampus (Fig. 1b; Mann-Whitney test, $p>0.05$ ).

We performed Western blot assay to confirm actin downregulation in DG neurons. We observed a $\sim 50 \%$ drop in $\beta$-actin protein expression levels in the DG in SRF-deficient neurons (Fig. 1d, e). In non-neuronal cells, the downregulation of $\beta$-actin induces cofilin 1 phosphorylation at Ser3, leading to its inhibition (Liu et al. 2007). Cofilin 1 is an actin-binding protein that regulates actin filament dynamics, stimulating the severance of actin filaments. To test whether the low level of $\beta$-actin and $\gamma$-actin in adult neurons correlates with changes in the actin-binding proteins, we examined the expression of cofilin 1 in the DG. Western blot analysis revealed that cofilin 1 phosphorylation was upregulated in SRF KO DG extracts (Fig. 1f) and coincided with a lower level of $\beta$-actin (Fig. 1d).

\section{Morphology of dendritic spines in granular neurons that lack SRF}

Actin dynamics play a crucial role in neurons, especially in the regulation of dendritic spine morphology (Hotulainen and Hoogenraad 2010; Hotulainen et al. 2009). Therefore, we investigated whether a decrease in $\beta$-actin protein expression and increase in cofilin 1 phosphorylation affect dendritic spine morphology. We evaluated the effects of SRF deficiency on the density and morphology of dendritic spines of GCs in the molecular layer of the dorsal DG (Fig. 2a). The analysis of spine density showed no significant changes in the number of spines of DG neurons (upper blade; Fig. 2b; $t$ test, $p>0.05$ ). To assess whether SRF depletion modified dendritic spine morphology, we measured the length and width of the spines to evaluate spine shape (Michaluk et al. 2011). The cumulative probability graph shows a significant increase in length-to-width ratio in DG neurons from SRF KO animals (Fig. 2c; Kolmogorov-Smirnov test, $p<0.001$ ) which testify change towards longer and thinner dendritic spines. To further investigate the way in which SRF depletion affects dendritic spine shape, we clustered spines into two morphological categories: long spines vs. mushroom and stubby spines (Fig. 2d) (Jasinska et al. 2016). Serum 


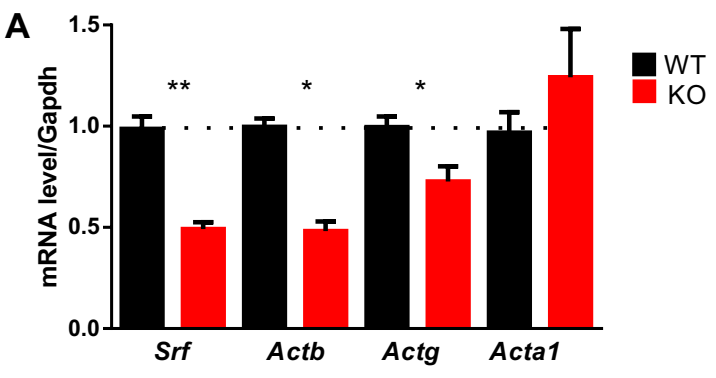

B

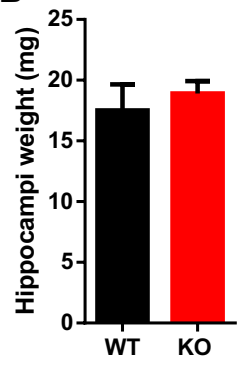

C

\section{D}

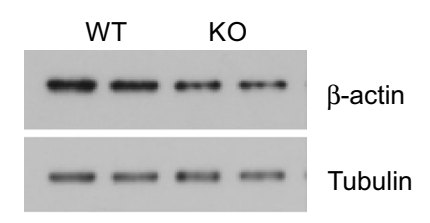

$\mathbf{F}$

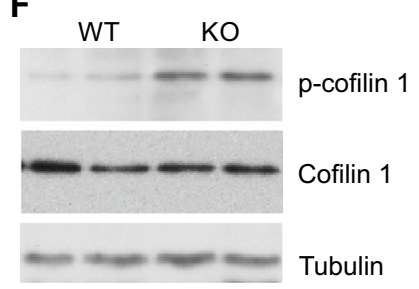

E
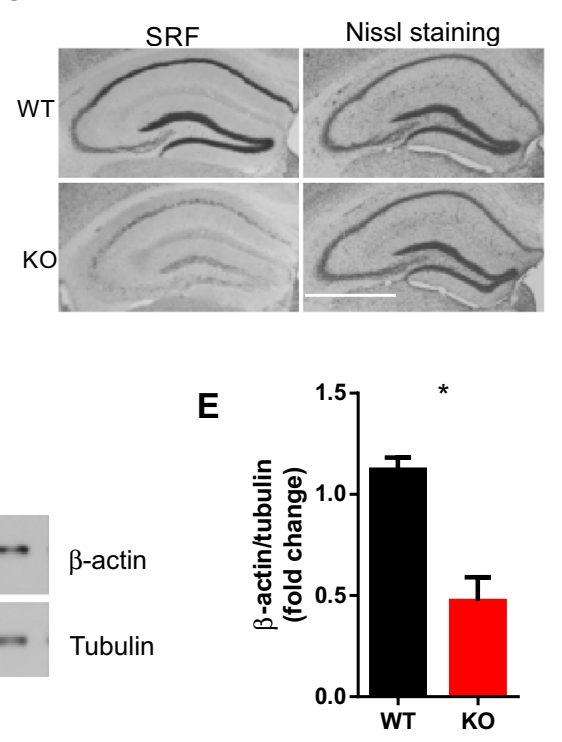

G

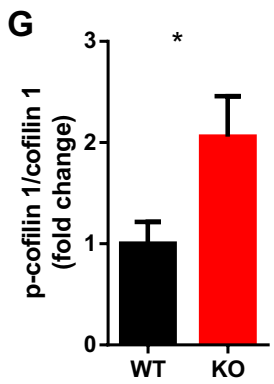

Fig. 1 The lack of SRF does not affect the general architecture of the adult hippocampus despite changes of actin cytoskeletal components. a Quantitative real-time RT-PCR analysis of Srf, Actb, and Actg and Actal mRNA levels in the hippocampus in control wild-type littermates (WT) and knockout mice (KO; $n \geq 4$ for each genotype; MannWhitney nonparametric test, SRF, $* * p=0.0079$; Actb, $* * p=0.0079$, Actg, ${ }^{*} p=0.0159$, Acta1, $p=0.5556$, ns). b Hippocampus weight analysis in control and SRF KO animals ( $n=6$ for each genotype; Mann-Whitney nonparametric test, $p=0.3000$, ns). No differences in total hippocampus weight were found between genotypes. c Nissl staining of brain sections from WT and SRF KO animals. d Western blot analysis with $\beta$-actin antibody. Each line represents a single animal. e Downregulation of $\beta$-actin protein in DG extracts from SRF KO animals $\left(n \geq 4\right.$; Mann-Whitney nonparametric test, $\left.{ }^{*} p=0.0159\right)$. f Western blot analysis with p-cofilin 1 and cofilin 1 antibody. Each line represents a single animal. $g$ Increase in cofilin 1 phosphorylation in DG extracts from SRF KO mice relative to total cofilin 1 level ( $n \geq 4$; Mann-Whitney nonparametric test, ${ }^{*} p=0.0175$ )

response factor $\mathrm{KO}$ mice exhibited a significant increase $(\sim 6$ percentage points) in the frequency of long spines in the DG and a significant decrease in the population of mushroom and stubby spines $\left(\chi^{2}\right.$ test, $\left.p<0.0001\right)$ compared with WT animals.

The morphology of dendritic spines under many conditions is highly correlated with changes in synaptic strength (Engert and Bonhoeffer 1999; Kasai et al. 2003; Yuste and Bonhoeffer 2001). To test whether SRF deletion affects basal glutamatergic transmission in adult neurons, $\alpha$-amino3-hydroxy-5-methyl-4-isoxazolepropionic acid (AMPA) receptor-mediated miniature excitatory postsynaptic currents (mEPSCs) were measured (Fig. 3). The comparison of cumulative probability of cellular responses between WT mice and SRF KO mice demonstrated a significant decrease in mEPSC amplitude (Fig. 3b; Kolmogorov-Smirnov test, $p<0.001$ ) and frequency (Fig. 3d; Kolmogorov-Smirnov test, $p=0.01$ ).

\section{Serum response factor deletion in mature neurons induces hyperactivity and alterations of species-typical behaviors}

To determine whether changes in the morphology and function of DG neurons in SRF KO animals correlate with behavioral deficits, we compared locomotor activity in WT and SRF KO animals in the open field test. Serum response factor $\mathrm{KO}$ animals were significantly more active than WT animals during a 15-min exploration session (Fig. 4a). Differences between genotypes were apparent from the first minute of exploration until the end of the test session (Fig. 4b; repeated-measures analysis of variance [ANOVA], genotype: $F_{1,11}=25.63, p=0.0004$, time: $F_{4,44}=21.28$, $p<0.0001$, genotype $\times$ time: $F_{4,44}=0.1032, p=0.9808, \mathrm{~ns}$ ).

Next, we evaluated the role of SRF using simple, hippocampus-dependent tasks, including digging, marble burying, and nest building (Deacon 2006; Deacon et al. 2002). In the digging paradigm, SRF KO animals performed fewer digging bouts (Fig. 5a; Mann-Whitney test, $p<0.05$ ). Serum response factor $\mathrm{KO}$ animals also spent less time digging than control animals (Fig. 5b; Mann-Whitney test, $p<0.01$ ). No difference in the latency to start digging was found between KO and WT animals (Fig. 5c; Mann-Whitney test, $p>0.05$ ). In the marble burying test, SRF KO animals exhibited deficits, in which they buried fewer marbles than WT littermate controls (Fig. 5d, e; Mann-Whitney test, $p<0.01$ ). Finally, SRF KO mice exhibited impairments in nest building compared with WT littermates (Fig. 5f, g; Mann-Whitney test, $p<0.01)$. 
Fig. 2 Serum response factor expression in mature excitatory neurons is essential for maintaining the proper structure of dendritic spines. a Example photographs of DiI-stained dendrites with dendritic spines of granule neurons from the dentate gyrus in WT and SRF $\mathrm{KO}$ animals ( $n=8$ animals, males and females). b Dendritic spine density of DG neurons in WT and SRF KO animals $(t$ test, $p=0.6374$, ns). c Cumulative histogram of dendritic spine length-to-width ratio $(\log )$ in WT and SRF KO DG neurons. SRF KO mice had a higher length-to-width ratio than WT mice (Kolmogorov-Smirnov test, $* p=0.0244)$. d Percentage of protrusions clustered into two categories (long spines vs. mushroom and stubby spines) in the DG $\left(\chi^{2}=24, d f=29.1\right.$, $p<0.001)$. Dendritic spine number and morphology were analyzed using semiautomated SpineMagic! software. Spine clustering was performed using custom scripts
A

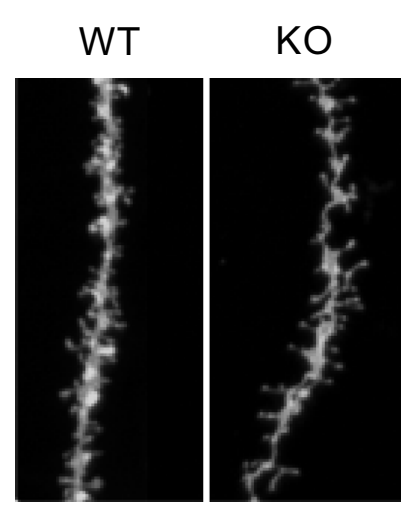

C

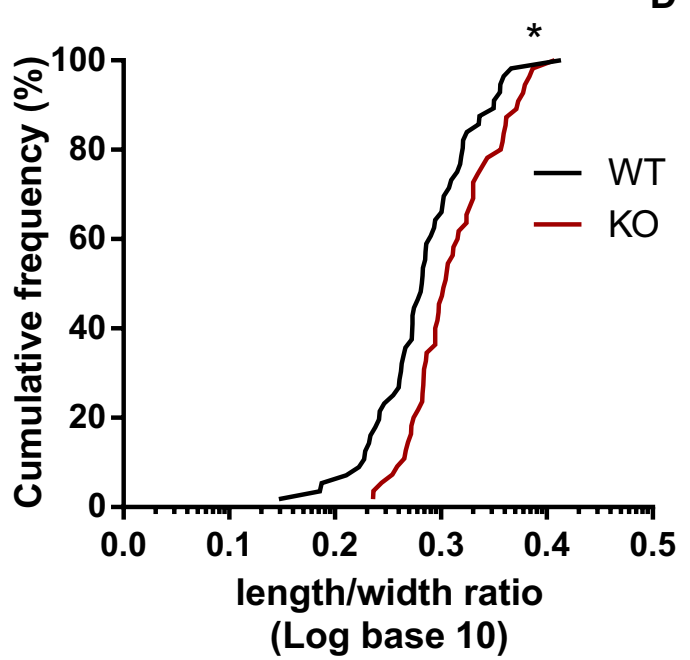

B

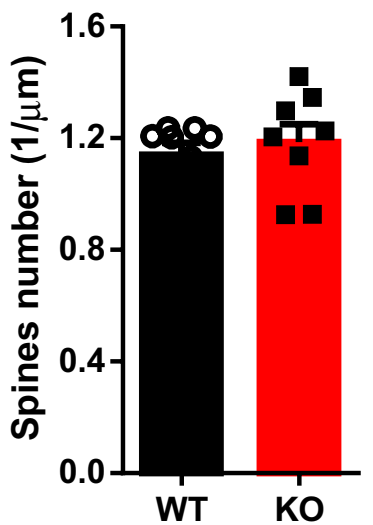

D

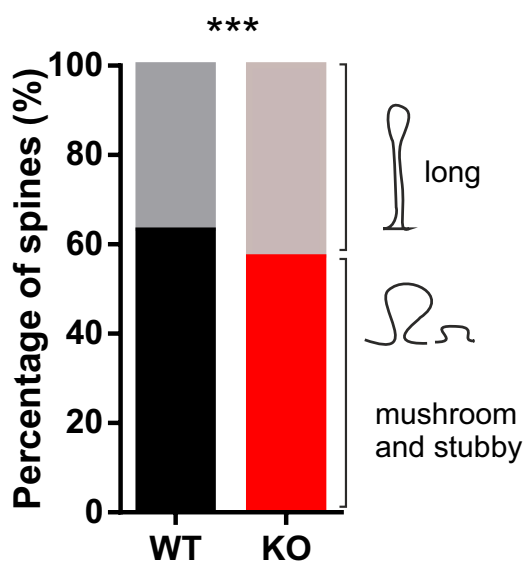

\section{Discussion}

The present study assessed morphological, electrophysiological, and behavioral effects of SRF deficiency in adult excitatory granular neurons. The depletion of SRF in adult neurons leds to the direct downregulation of mRNAs of two actin-encoding genes and the functional inhibition of cofilin 1. Although alterations of actins and actin-binding protein did not alter gross hippocampal architecture, changes in dendritic spine morphology and basal synaptic transmission in granular neurons of the DG were observed. Moreover, SRF KO animals exhibited hyperactivity and impairments in behaviors that are typical for small rodents, recognized as hippocampus-dependent tasks. Altogether, our results suggest that basal SRF expression in adult DG neurons is engaged in the regulation of structural plasticity, basal synaptic transmission, and species-typical behaviors.

Alterations of spine morphology without gross changes in hippocampal architecture were the most prominent phenotype we identified in animals with a decrease in SRF expression in adult neurons. At the molecular level, we observed a decrease in the expression of neuronal actins and an increase in the phosphorylation of the actin-depolymerization factor cofilin 1 . The actin cytoskeleton in neurons is composed of two distinct isoforms, non-muscle $\gamma$-actin and $\beta$-actin. Both actin genes are SRF targets and are downregulated in DG neurons in SRF KO animals (Miano et al. 2007; Sun et al. 2006). The present results confirmed earlier findings. The expression of both $\beta$-actin and $\gamma$-actin is impaired upon SRF ablation. We essentially generated the double knockdown of brain-expressed actin isoforms in $\mathrm{Srf}$ mouse mutants.

We also showed that the depletion of SRF in adult neurons coincided with an increase in cofilin 1 phosphorylation at Ser3 (inactivation), suggesting that in wild-type cells, SRF is involved in cofilin activation. Cofilin 1 activity could be inhibited by increased phosphorylation by LIM kinases (LIMKs) or decreased activity of phosphatases like slingshot (SSH) family of protein phosphatases (Mizuno 2013). In MRTFs-deficient neurons (myocardin-related transcription factors; SRF coactivators), a decrease in Pctaire1 kinase activity was shown to stimulate the Pak1-Limk cascade to promote cofilin phosphorylation (Mokalled et al. 2010). Pctaire 1 (Cdk16) gene is a direct target of SRF/MRTF (Mokalled et al. 2010). Moreover, the decrease in actin 
A

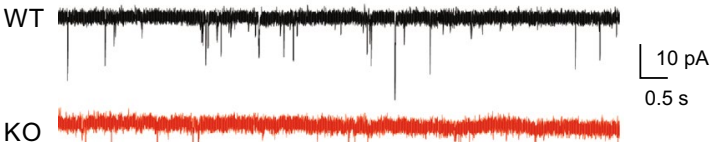

B

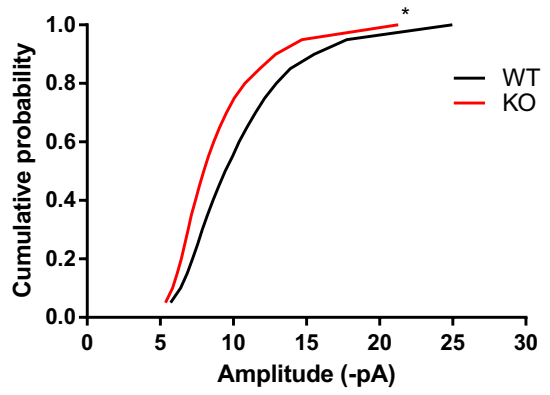

C

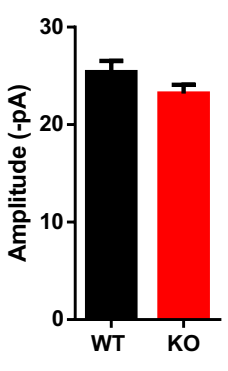

D
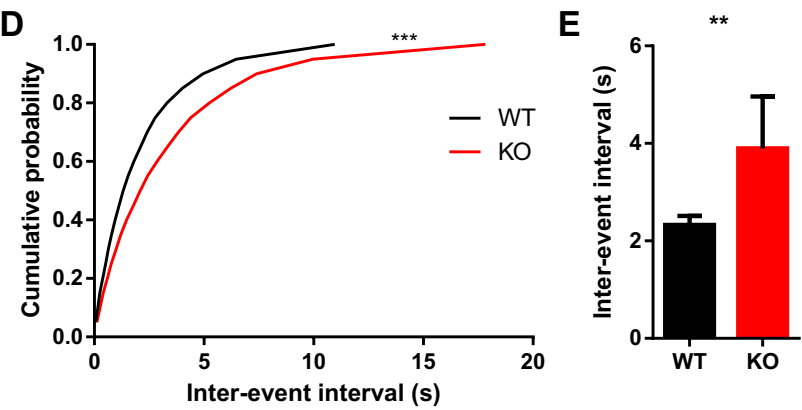

Fig. 3 Reduction of the amplitude and frequency of spontaneous mEPSCs in SRF KO granular neurons. a Sample traces from WT and SRF KO granular neurons. Cumulative probability plots showing mEPSCs amplitude (b) and frequency (d) in $n=16$ WT cells and $n=14$ SRF KO neurons (Kolmogorov-Smirnov test, $* p=0.01$ and $* * * p<0.001$, respectively). Bar graphs in $\mathbf{c}$ and e represent averaged amplitudes and frequencies of mEPSCs (Mann-Whitney test, $p=0.1246$, ns and ${ }^{* *} p=0.003$, respectively)

levels observed in SRF-deficient neurons might impair slingshot activation, which is stimulated by association with actin filaments (Kurita et al. 2008; Liu et al. 2007; Nagata-Ohashi et al. 2004). Both mechanisms of increased cofilin 1 phosphorylation observed in SRF-deficient neurons are possible. Collectively, our data are consistent with a previous study that reported higher levels of phosphorylated cofilin 1 in SRF- or MRTFs -depleted neurons (Alberti et al. 2005; Beck et al. 2012; Mokalled et al. 2010; Zimprich et al. 2017).

In the present study, we observed a sharp reduction of total actin levels in SRF-depleted neurons. Interestingly, SRF deletion in non-neuronal cells resulted in the downregulation of $\beta$-actin and a decrease in the ratio of polymerized F-actin to G-actin (Randrianarison-Huetz et al. 2018; Schratt et al. 2002; Taylor et al. 2014). Thus, changes in spine morphology in SRF-depleted neurons could be attributable to both lower total actin expression and alterations of the regulation of actin polymerization. However, further quantification of the F-actin-to-G-actin ratio in neurons is needed. The link between SRF and actin is multifaceted. Actin is a direct target of SRF and controls SRF-dependent gene expression (Sotiropoulos et al. 1999). The conversion of monomeric G-actin to polymeric F-actin results in the activation of SRF-dependent transcription that is regulated by the translocation of MRTFs (Kalita et al. 2006; Kaneda et al. 2018) from the cytoplasm to the nucleus (Miralles et al. 2003; Posern et al. 2002, 2004). Moreover, nuclear actin dynamics were also shown to regulate the activity of SRF/ MRTFs (Baarlink et al. 2013; Ho et al. 2013; Lundquist et al. 2014; Vartiainen et al. 2007). Thus, there is a feedback loop between SRF and actin, in which the activity of SRF regulates actin levels, the dynamics of which influence the transcriptional activity of SRF.

Actin cytoskeleton remodeling, stabilization, or depolymerization is crucial for dendritic spine morphology and synaptic function (Ethell and Pasquale 2005; Honkura et al. 2008; Matus 2000). Modifications of the number of neuronal connections and the size and shape of dendritic spines underlie synaptic plasticity (Borczyk et al. 2019; Holtmaat and Svoboda 2009; Kasai et al. 2010). Alterations of spine morphology underlie many neuropsychiatric disorders, indicating their functional importance (Glausier and Lewis 2013; Penzes et al. 2011; Phillips and Pozzo-Miller 2015). Spine parameters, such as the dimension of the spine head or neck, determine spine motility and stability (Nimchinsky et al. 2002). Mushroom-shaped spines are generally considered mature and stable. In contrast, thin spines with a long neck are highly plastic (Bourne and Harris 2007). In the present study, we found that SRF depletion in granular neurons shifted the spine population toward long spines with a less mature phenotype. Thus, dendritic spines grew but did not form enlarged heads, which are characteristic of mature mushroom spines. In addition to spine morphology, we observed deficits in basal synaptic transmission in SRFdepleted neurons. The lower amplitude (mostly mEPSCs of small and medium amplitudes) and frequency of mEPSCs that were observed in DG neurons that lacked SRF could represent either a change in release probability or postsynaptic processes, such as the loss of functional synapses.

Using several behavioral tasks, we showed that animals that lacked SRF protein in adult forebrain neurons built poorer nests, performed fewer digging bouts, exhibited a reduction of burying behavior compared with their WT littermates. In agreement with other studies, mice lacking SRF were significantly more active than control animals in the open field test (Johnson et al. 2011; Parkitna et al. 2010; Zimprich et al. 2017). Similar alterations in innate behaviors and activity were observed in animals with hippocampal lesions (Deacon et al. 2002). Deficits in nest building, digging and marble burying, in SRF KO animals, were also present in the animal models with smaller 
A

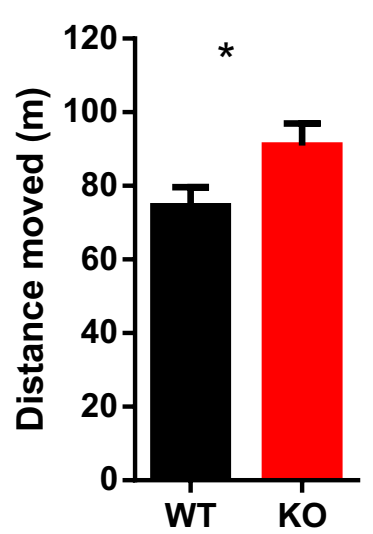

B

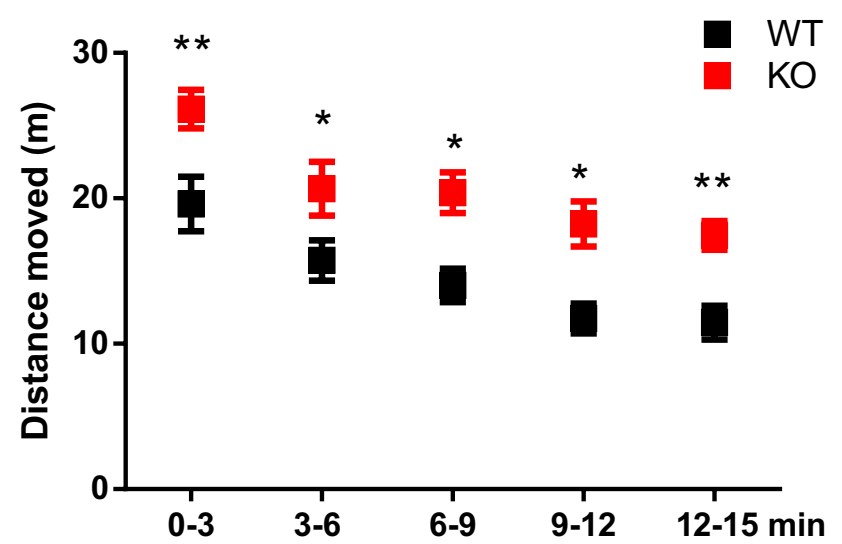

Fig. 4 SRF KO mice exhibit increase in spontaneous activity in the open field test. Mice individually explored the open field for $15 \mathrm{~min}$. a Distance traveled in $15 \mathrm{~min}$ by WT and SRF KO mice $n=12$ WT, $n=12$ SRF KO female adult animals ( $t$ test, $* p=0.0484)$. b Mutant animals were significantly more active in all 3-min time bins in the open field (two-way repeated-measures ANOVA: genotype: $F_{1,11}=25.63, p=0.0004$, time: $F_{4,44}=21.28, p<0.0001$, genotype $\times$ time: $F_{4,44}=0.1032, p=0.9808$; followed by Sidak multiplecomparison post hoc test: $* p<0.05, * * p<0.01$ )

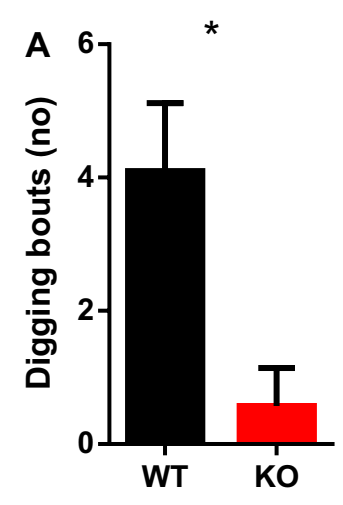

D

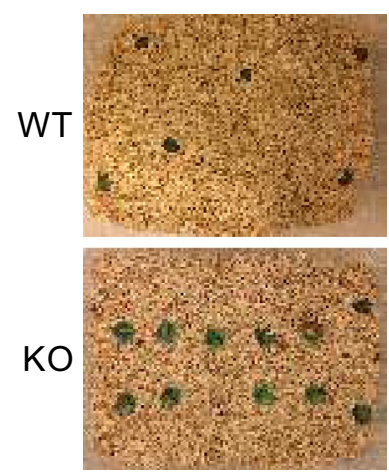

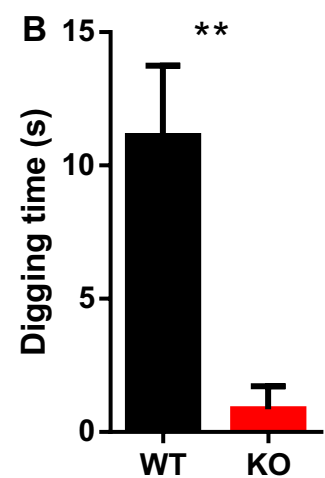

$\mathbf{F}$

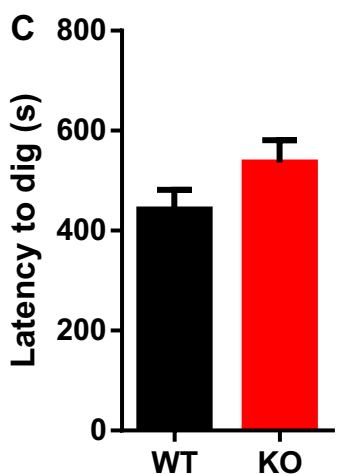

G

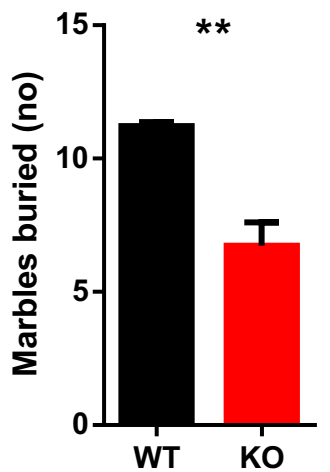

$\mathrm{KO}$

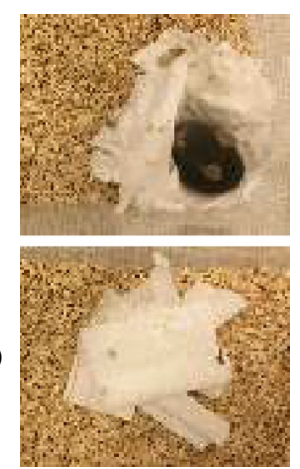

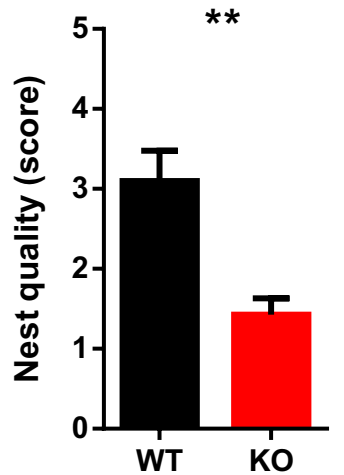

Fig. 5 Non-cognitive-dependent behaviors are impaired in SRF KO animals. Species-typical behavioral tasks were analyzed $(n=10 \mathrm{WT}$, $n=7$ SRF KO). a SRF KO mice made fewer digging bouts (MannWhitney $t$ test, $* p=0.0154)$. b The time spent digging was shorter in SRF KO mice (Mann-Whitney $t$ test, ${ }^{* *} p=0.0086$ ). c Latency to start digging did not differ between genotypes (Mann-Whitney $t$ test, $p=0.1748, \mathrm{~ns})$. d Photographs of cages after 30-min session. e The number of marbles that were buried was less in SRF KO mice than in WT controls (Mann-Whitney $t$ test, ${ }^{*} p=0.0047$ ). f Photographs of cages after nest building test. g SRF KO animals built less complex nests than WT animals (Mann-Whitney $t$ test, ${ }^{* *} p=0.0070$ ) 
volume of the DG and abnormal spine morphology of DG neurons (Kondratiuk et al. 2013, 2017) and in mice with deficits in DGs adult neurogenesis (Jedynak et al. 2012). Although behaviors observed in SRF KO animals may reflect dysfunction of the dentate gyrus, we cannot exclude the possibility that SRF deletion in other brain structures impacts the observed phenotype. Interestingly, marble burying and nest building tests are interpreted by some authors as a measure of repetitive or social behaviors, respectively, were found to be altered in several murine models of psychiatric disorders, such as schizophrenia and autism spectrum disorders (Crawley 2012; Deacon 2006; Deacon et al. 2002; Jirkof 2014).

Loss-of-function of the ACTB gene in humans is associated with developmental delays or intellectual disability (Cuvertino et al. 2017; Palumbo et al. 2017). Moreover, a single-nucleotide polymorphism (SNP) of the ACTB gene that caused missense mutations was shown to be linked with several neurological phenotypes, including global developmental delay, intellectual disability, cognitive impairment, abnormal aggressive and impulsive behavior, attention deficit, hyperactivity, and autism spectrum disorders (based on DECIPHER database; http://www.decipher.sanger.ac.uk/, accessed 2018). An increase in hyperactivity and deficits in species-typical behaviors in SRF KO animals also resemble the phenotype of mice with the brain-specific deletion of $\beta$-actin, potentially linking some of the observed phenotypes in SRF-deficient neurons to the specific deletion of $\beta$-actin (Cheever et al. 2012). However, further work is needed to determine whether the specific depletion of $\beta$-actin and $\gamma$-actin in adult neurons mimics the phenotype that we observed in SRF KO animals. Overall, the present data support the notion that such behaviors as nest building, marble burying, and locomotor activity require intact hippocampal circuity.

Several lines of evidence suggest a role for SRF in brain pathology, such as epileptogenesis, cocaine-induced gene expression, and cocaine-induced dendritic spine formation (Cahill et al. 2017; Kuzniewska et al. 2016; Losing et al. 2017; Parkitna et al. 2010). Data also link SNPs of the $S r f$ and Mrtfs genes to brain diseases. In humans, SNPs in CArG box (SRF transcription factor binding site), that disrupt SRF binding, were linked with neurological disorders, such as bipolar disorder, amyotrophic lateral sclerosis, and Alzheimer's disease (Benson et al. 2011). Moreover, SNPs of the $M r t f s$ genes were associated with neurodevelopmental disorders, such as schizophrenia and autism spectrum disorders (Holt et al. 2010; Luo et al. 2015; Wang et al. 2016).

Altogether, our data indicate that the adult deletion of SRF in DG neurons alters the expression of actins, dendritic spine morphology, and mouse behavior, showing that SRF expression is necessary to maintain proper brain circuitry. To our knowledge, this is the first report that demonstrates that SRF regulates the adult structural plasticity of intact, adult hippocampal neurons in vivo. Furthermore, the present results link aberrant spine morphology to changes in glutamatergic synaptic transmission and behavior.

Acknowledgements This work was supported by the Polish National Science Centre grant (SONATA BIS 2) DEC-2012/07/E/NZ3/01814.

Author contributions $\mathrm{KN}, \mathrm{AK}, \mathrm{AB}, \mathrm{MS}$, MP, and $\mathrm{BK}$ performed the experiments and analyzed the data. KR designed the behavioral experiments. LK discussed the data. KK supervised and designed the experiments and wrote the paper.

\section{Compliance with ethical standards}

Conflict of interest The authors declare that they have no conflict of interest.

Ethical approval All applicable international, national, and/or institutional guidelines for the care and use of animals were followed.

Informed consent No human subject was used in this study

Open Access This article is distributed under the terms of the Creative Commons Attribution 4.0 International License (http://creativeco mmons.org/licenses/by/4.0/), which permits unrestricted use, distribution, and reproduction in any medium, provided you give appropriate credit to the original author(s) and the source, provide a link to the Creative Commons license, and indicate if changes were made.

\section{References}

Alberti S et al (2005) Neuronal migration in the murine rostral migratory stream requires serum response factor. Proc Natl Acad Sci USA 102:6148-6153

Amaral DG, Scharfman HE, Lavenex P (2007) The dentate gyrus: fundamental neuroanatomical organization (dentate gyrus for dummies). Prog Brain Res 163:3-22. https://doi.org/10.1016/s0079 $-6123(07) 63001-5$

Baarlink C, Wang H, Grosse R (2013) Nuclear actin network assembly by formins regulates the SRF coactivator MAL. Science 340:864867. https://doi.org/10.1126/science. 1235038

Beck H, Flynn K, Lindenberg KS, Schwarz H, Bradke F, Di Giovanni S, Knoll B (2012) Serum Response Factor (SRF)-cofilin-actin signaling axis modulates mitochondrial dynamics. Proc Natl Acad Sci USA 109:E2523-E2532. https://doi.org/10.1073/pnas.12081 41109

Benito E, Valor LM, Jimenez-Minchan M, Huber W, Barco A (2011) cAMP response element-binding protein is a primary hub of activity-driven neuronal gene expression. J Neurosci 31:18237-18250

Benson CC, Zhou Q, Long X, Miano JM (2011) Identifying functional single nucleotide polymorphisms in the human CArGome. Physiol Genomics 43:1038-1048

Borczyk M, Sliwinska MA, Caly A, Bernas T, Radwanska K (2019) Neuronal plasticity affects correlation between the size of dendritic spine and its postsynaptic density. Sci Rep 9:1693. https:// doi.org/10.1038/s41598-018-38412-7

Bourne J, Harris KM (2007) Do thin spines learn to be mushroom spines that remember? Curr Opin Neurobiol 17:381-386. https:// doi.org/10.1016/j.conb.2007.04.009 
Cahill ME et al (2017) The dendritic spine morphogenic effects of repeated cocaine use occur through the regulation of serum response factor signaling. Mol Psychiatry. https://doi.org/10.1038/ mp.2017.116

Cheever TR, Li B, Ervasti JM (2012) Restricted morphological and behavioral abnormalities following ablation of beta-actin in the brain. PLoS One 7:e32970. https://doi.org/10.1371/journ al.pone. 0032970

Crawley JN (2012) Translational animal models of autism and neurodevelopmental disorders. Dialogues Clin Neurosci 14:293-305

Cuvertino $S$ et al (2017) ACTB loss-of-function mutations result in a pleiotropic developmental disorder. Am J Hum Genet 101:10211033. https://doi.org/10.1016/j.ajhg.2017.11.006

Deacon RM (2006) Digging and marble burying in mice: simple methods for in vivo identification of biological impacts. Nat Protoc 1:122-124. https://doi.org/10.1038/nprot.2006.20

Deacon RM, Rawlins JN (2005) Hippocampal lesions, species-typical behaviours and anxiety in mice. Behav Brain Res 156:241-249. https://doi.org/10.1016/j.bbr.2004.05.027

Deacon RM, Croucher A, Rawlins JN (2002) Hippocampal cytotoxic lesion effects on species-typical behaviours in mice. Behav Brain Res 132:203-213

Engert F, Bonhoeffer T (1999) Dendritic spine changes associated with hippocampal long-term synaptic plasticity. Nature 399:66-70. https://doi.org/10.1038/19978

Erdmann G, Schutz G, Berger S (2007) Inducible gene inactivation in neurons of the adult mouse forebrain. BMC Neurosci 8:63

Ethell IM, Pasquale EB (2005) Molecular mechanisms of dendritic spine development and remodeling. Prog Neurobiol 75:161-205. https://doi.org/10.1016/j.pneurobio.2005.02.003

Etkin A, Alarcon JM, Weisberg SP, Touzani K, Huang YY, Nordheim A, Kandel ER (2006) A role in learning for SRF: deletion in the adult forebrain disrupts LTD and the formation of an immediate memory of a novel context. Neuron 50:127-143

Glausier JR, Lewis DA (2013) Dendritic spine pathology in schizophrenia. Neuroscience 251:90-107. https://doi.org/10.1016/j. neuroscience.2012.04.044

Hagihara H, Takao K, Walton NM, Matsumoto M, Miyakawa T (2013) Immature dentate gyrus: an endophenotype of neuropsychiatric disorders. Neural Plast 2013:318596. https://doi. org/10.1155/2013/318596

Ho CY, Jaalouk DE, Vartiainen MK, Lammerding J (2013) Lamin $\mathrm{A} / \mathrm{C}$ and emerin regulate MKL1-SRF activity by modulating actin dynamics. Nature 497:507-511. https://doi.org/10.1038/ nature 12105

Holt $\mathrm{R}$ et al (2010) Linkage and candidate gene studies of autism spectrum disorders in European populations. Eur J Hum Genet 18:1013-1019. https://doi.org/10.1038/ejhg.2010.69

Holtmaat A, Svoboda K (2009) Experience-dependent structural synaptic plasticity in the mammalian brain. Nat Rev Neurosci 10:647-658. https://doi.org/10.1038/nrn2699

Honkura N, Matsuzaki M, Noguchi J, Ellis-Davies GC, Kasai H (2008) The subspine organization of actin fibers regulates the structure and plasticity of dendritic spines. Neuron 57:719-729. https://doi. org/10.1016/j.neuron.2008.01.013

Hotulainen P, Hoogenraad CC (2010) Actin in dendritic spines: connecting dynamics to function. J Cell Biol 189:619-629. https:// doi.org/10.1083/jcb.201003008

Hotulainen P, Llano O, Smirnov S, Tanhuanpaa K, Faix J, Rivera C, Lappalainen P (2009) Defining mechanisms of actin polymerization and depolymerization during dendritic spine morphogenesis. J Cell Biol 185:323-339. https://doi.org/10.1083/jcb.200809046

Jasinska M, Milek J, Cymerman IA, Leski S, Kaczmarek L, Dziembowska M (2016) miR-132 Regulates dendritic spine structure by direct targeting of matrix metalloproteinase 9 mRNA. Mol Neurobiol 53:4701-4712. https://doi.org/10.1007/s12035-015-9383-Z
Jedynak P, Jaholkowski P, Wozniak G, Sandi C, Kaczmarek L, Filipkowski RK (2012) Lack of cyclin D2 impairing adult brain neurogenesis alters hippocampal-dependent behavioral tasks without reducing learning ability. Behav Brain Res 227(1):159-166

Jirkof P (2014) Burrowing and nest building behavior as indicators of well-being in mice. J Neurosci Methods 234:139-146. https://doi. org/10.1016/j.jneumeth.2014.02.001

Johnson AW, Crombag HS, Smith DR, Ramanan N (2011) Effects of serum response factor (SRF) deletion on conditioned reinforcement. Behav Brain Res 220:312-318. https://doi.org/10.1016/j. bbr.2011.02.015

Kalita K, Kharebava G, Zheng JJ, Hetman M (2006) Role of megakaryoblastic acute leukemia-1 in ERK1/2-dependent stimulation of serum response factor-driven transcription by BDNF or increased synaptic activity. J Neurosci 26:10020-10032

Kaneda M et al (2018) Synaptic localisation of SRF coactivators, MKL1 and MKL2, and their role in dendritic spine morphology. Sci Rep 8:727. https://doi.org/10.1038/s41598-017-18905-7

Kasai H, Matsuzaki M, Noguchi J, Yasumatsu N, Nakahara H (2003) Structure-stability-function relationships of dendritic spines. Trends Neurosci 26:360-368. https://doi.org/10.1016/s0166 -2236(03)00162-0

Kasai H, Fukuda M, Watanabe S, Hayashi-Takagi A, Noguchi J (2010) Structural dynamics of dendritic spines in memory and cognition. Trends Neurosci 33:121-129. https://doi. org/10.1016/j.tins.2010.01.001

Kempermann G, Jessberger S, Steiner B, Kronenberg G (2004) Milestones of neuronal development in the adult hippocampus. Trends Neurosci 27:447-452. https://doi.org/10.1016/j. tins.2004.05.013

Knoll B, Kretz O, Fiedler C, Alberti S, Schutz G, Frotscher M, Nordheim A (2006) Serum response factor controls neuronal circuit assembly in the hippocampus. Nat Neurosci 9:195-204

Kondratiuk I, Devijver H, Lechat B, Van Leuven F, Kaczmarek L, Filipkowski RK (2013) Glycogen synthase kinase-3beta affects size of dentate gyrus and species-typical behavioral tasks in transgenic and knockout mice. Behav Brain Res 248:46-50

Kondratiuk I, Łęski S, Urbańska M, Biecek P, Devijver H, Lechat B, Van Leuven F, Kaczmarek L, Jaworski T (2017) GSK-3 $\beta$ and MMP-9 cooperate in the control of dendritic spine morphology. Mol Neurobiol 54(1):200-211

Kurita S, Watanabe Y, Gunji E, Ohashi K, Mizuno K (2008) Molecular dissection of the mechanisms of substrate recognition and F-actin-mediated activation of cofilin-phosphatase Slingshot-1. J Biol Chem 283:32542-32552. https://doi.org/10.1074/jbc. m804627200

Kuzniewska B, Nader K, Dabrowski M, Kaczmarek L, Kalita K (2016) Adult deletion of SRF increases epileptogenesis and decreases activity-induced gene expression. Mol Neurobiol 53:1478-1493. https://doi.org/10.1007/s12035-014-9089-7

Liu $\mathrm{N}$ et al (2007) Actin deficiency induces cofilin phosphorylation: proteome analysis of HeLa cells after beta-actin gene silencing. Cell Motil Cytoskeleton 64:110-120. https://doi.org/10.1002/ cm.20169

Losing P et al (2017) SRF modulates seizure occurrence, activity induced gene transcription and hippocampal circuit reorganization in the mouse pilocarpine epilepsy model. Mol Brain 10:30. https://doi.org/10.1186/s13041-017-0310-2

Lu PP, Ramanan N (2011) Serum response factor is required for cortical axon growth but is dispensable for neurogenesis and neocortical lamination. J Neurosci 31:16651-16664

Lundquist MR, Storaska AJ, Liu TC, Larsen SD, Evans T, Neubig RR, Jaffrey SR (2014) Redox modification of nuclear actin by MICAL-2 regulates SRF signaling. Cell 156:563-576. https:// doi.org/10.1016/j.cell.2013.12.035 
Luo XJ, Huang L, Oord EJ, Aberg KA, Gan L, Zhao Z, Yao YG (2015) Common variants in the MKL1 gene confer risk of schizophrenia. Schizophr Bull 41:715-727. https://doi.org/10.1093/ schbul/sbu 156

Matus A (2000) Actin-based plasticity in dendritic spines. Science 290:754-758

Miano JM, Long X, Fujiwara K (2007) Serum response factor: master regulator of the actin cytoskeleton and contractile apparatus. Am J Physiol Cell Physiol 292:C70-C81

Michaluk $P$ et al (2011) Influence of matrix metalloproteinase MMP-9 on dendritic spine morphology. J Cell Sci 124:3369-3380

Miralles F, Posern G, Zaromytidou AI, Treisman R (2003) Actin dynamics control SRF activity by regulation of its coactivator MAL. Cell 113:329-342

Mizuno K (2013) Signaling mechanisms and functional roles of cofilin phosphorylation and dephosphorylation. Cell Signal 25:457-469. https://doi.org/10.1016/j.cellsig.2012.11.001

Mokalled MH, Johnson A, Kim Y, Oh J, Olson EN (2010) Myocardinrelated transcription factors regulate the $\mathrm{Cdk} 5 / \mathrm{Pctaire} 1$ kinase cascade to control neurite outgrowth, neuronal migration and brain development. Development 137:2365-2374

Moretti P, Adriaan Bouwknecht J, Teague R, Paylor R, Zoghbi HY (2005) Abnormalities of social interactions and home-cage behavior in a mouse model of Rett syndrome. Hum Mol Genet 14(2):205-220

Nagata-Ohashi K et al (2004) A pathway of neuregulin-induced activation of cofilin-phosphatase Slingshot and cofilin in lamellipodia. J Cell Biol 165:465-471. https://doi.org/10.1083/jcb.200401136

Nimchinsky EA, Sabatini BL, Svoboda K (2002) Structure and function of dendritic spines. Annu Rev Physiol 64:313-353. https:// doi.org/10.1146/annurev.physiol.64.081501.160008

Palumbo O et al (2017) Refinement of the critical 7p22.1 deletion region: Haploinsufficiency of ACTB is the cause of the $7 \mathrm{p} 22.1$ microdeletion-related developmental disorders. Eur J Med Genet. https://doi.org/10.1016/j.ejmg.2017.12.008

Parkitna JR et al (2010) Loss of the serum response factor in the dopamine system leads to hyperactivity. Faseb J 24:2427-2435

Penzes P, Cahill ME, Jones KA, VanLeeuwen JE, Woolfrey KM (2011) Dendritic spine pathology in neuropsychiatric disorders. Nat Neurosci 14:285-293. https://doi.org/10.1038/nn.2741

Phillips M, Pozzo-Miller L (2015) Dendritic spine dysgenesis in autism related disorders. Neurosci Lett 601:30-40. https://doi. org/10.1016/j.neulet.2015.01.011

Posern G, Sotiropoulos A, Treisman R (2002) Mutant actins demonstrate a role for unpolymerized actin in control of transcription by serum response factor. Mol Biol Cell 13:4167-4178

Posern G, Miralles F, Guettler S, Treisman R (2004) Mutant actins that stabilise F-actin use distinct mechanisms to activate the SRF coactivator MAL. EMBO J 23:3973-3983

Radic T, Friess L, Vijikumar A, Jungenitz T, Deller T, Schwarzacher SW (2017) Differential postnatal expression of neuronal maturation markers in the dentate gyrus of mice and rats. Front Neuroanat 11:104. https://doi.org/10.3389/fnana.2017.00104

Ramanan N, Shen Y, Sarsfield S, Lemberger T, Schutz G, Linden DJ, Ginty DD (2005) SRF mediates activity-induced gene expression and synaptic plasticity but not neuronal viability. Nat Neurosci 8:759-767

Randrianarison-Huetz V et al (2018) Srf controls satellite cell fusion through the maintenance of actin architecture. J Cell Biol 217:685-700. https://doi.org/10.1083/jcb.201705130

Ruszczycki B, Szepesi Z, Wilczynski GM, Bijata M, Kalita K, Kaczmarek L, Wlodarczyk J (2012) Sampling issues in quantitative analysis of dendritic spines morphology. BMC Bioinform 13:213

Scandaglia M et al (2015) Fine-tuned SRF activity controls asymmetrical neuronal outgrowth: implications for cortical migration, neural tissue lamination and circuit assembly. Sci Rep 5:17470. https:// doi.org/10.1038/srep17470

Schratt G, Philippar U, Berger J, Schwarz H, Heidenreich O, Nordheim A (2002) Serum response factor is crucial for actin cytoskeletal organization and focal adhesion assembly in embryonic stem cells. J Cell Biol 156:737-750. https://doi.org/10.1083/jcb.200106008

Sotiropoulos A, Gineitis D, Copeland J, Treisman R (1999) Signal-regulated activation of serum response factor is mediated by changes in actin dynamics. Cell 98:159-169

Stritt C, Knoll B (2010) Serum response factor regulates hippocampal lamination and dendrite development and is connected with reelin signaling. Mol Cell Biol 30:1828-1837

Sun Q, Chen G, Streb JW, Long X, Yang Y, Stoeckert CJ Jr, Miano JM (2006) Defining the mammalian CArGome. Genome Res 16:197-207. https://doi.org/10.1101/gr.4108706

Taylor A et al (2014) SRF is required for neutrophil migration in response to inflammation. Blood 123:3027-3036. https://doi. org/10.1182/blood-2013-06-507582

Treisman R (1995) Journey to the surface of the cell: fos regulation and the SRE. EMBO J 14:4905-4913

Vartiainen MK, Guettler S, Larijani B, Treisman R (2007) Nuclear actin regulates dynamic subcellular localization and activity of the SRF cofactor MAL. Science 316:1749-1752. https://doi. org/10.1126/science.1141084

Wang D et al (2016) Association between MKL1 rs6001946 and schizophrenia in a Han Chinese population. Neurosci Lett 631:36-39. https://doi.org/10.1016/j.neulet.2016.08.008

Yu DX, Marchetto MC, Gage FH (2014) How to make a hippocampal dentate gyrus granule neuron. Development 141:2366-2375. https ://doi.org/10.1242/dev.096776

Yuste R, Bonhoeffer T (2001) Morphological changes in dendritic spines associated with long-term synaptic plasticity. Annu Rev Neurosci 24:1071-1089. https://doi.org/10.1146/annurev.neuro 24.1.1071

Zimprich A et al (2017) Serum Response Factor (SRF) Ablation interferes with acute stress-associated immediate and long-term coping mechanisms. Mole Neurobiol 54:8242-8262. https://doi. org/10.1007/s12035-016-0300-x

Publisher's Note Springer Nature remains neutral with regard to jurisdictional claims in published maps and institutional affiliations. 